\title{
Racial disparities in pre-operative pain, function and disease activity for patients with rheumatoid arthritis undergoing Total knee or Total hip Arthroplasty: a New York based study
}

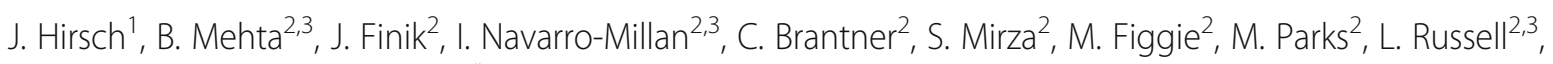

D. Orange $e^{2,4}$ and S. Goodman ${ }^{2,3^{*}}$ (D)

\begin{abstract}
Background: Black and Hispanic patients with osteoarthritis have more pain and worse function than Whites at the time of arthroplasty. Whether this is true for patients with rheumatoid arthritis (RA) is unknown.

Methods: This cross-sectional study used data on RA patients acquired between October 2013 and November 2018 prior to elective total knee (TKA) or hip arthroplasty (THA). Pain, function, and disease activity were assessed using the visual analogue scale (VAS), the Multidimensional Health Assessment Questionnaire (MDHAQ), and the Disease Activity Score (DAS28-ESR). We linked the cases to census tracts using geocoding to determine the community poverty level. Race, education, income, insurance and medications were collected via self-report. Using multivariable linear and logistic models we examined whether minority status predicted pain, function and RA disease activity at the time of arthroplasty.

Results: Thirty seven (23\%) of the 164 patients were Black or Hispanic (minorities). The MDHAQ and DAS28-ESR were not significantly worse while VAS pain score was significantly worse in minority patients $(p=0.03)$. There was no significant difference in education between the groups. Insurance varied significantly; $29 \%$ of minority patients had Medicaid vs. $0 \%$ of Whites $(p<0.0001)$. In the multivariable analyses minority status was not significantly associated with DAS28-ESR $[p=0.66]$, MDHAQ $[p=0.26]$, or VAS pain $[p=0.18]$.
\end{abstract}

Conclusions: For Black and/or Hispanic patients with RA undergoing THA or TKA at a high-volume specialty hospital, unlike Black or Hispanic patients with osteoarthritis (OA), there was no association with worse pain, function, or RA disease activity at the time of elective arthroplasty.

Keywords: Racial disparity, Minority groups, Rheumatoid arthritis (RA), Arthroplasty, Total knee arthroplasty (TKA), Total hip arthroplasty (THA), Pre-operative

\footnotetext{
* Correspondence: goodmans@hss.edu

${ }^{2}$ Hospital for Special Surgery, 535 E 70th Street, New York, NY 10021, USA

${ }^{3}$ Weill Cornell Medicine, New York, NY, USA

Full list of author information is available at the end of the article
}

C The Author(s). 2020 Open Access This article is distributed under the terms of the Creative Commons Attribution 4.0 International License (http://creativecommons.org/licenses/by/4.0/), which permits unrestricted use, distribution, and reproduction in any medium, provided you give appropriate credit to the original author(s) and the source, provide a link to the Creative Commons license, and indicate if changes were made. The Creative Commons Public Domain Dedication waiver (http://creativecommons.org/publicdomain/zero/1.0/) applies to the data made available in this article, unless otherwise stated. 


\section{Background}

Socioeconomic factors including race, poverty and educational attainment may affect multiple health outcomes, including arthroplasty utilization, and pain and function after arthroplasty [1]. Interactions between social factors may be complex. For instance, race and poverty are associated with worse arthroplasty outcomes, but education may mitigate the effect of poverty within poor communities [2]. In addition, worse arthroplasty outcomes associated with Black race are not seen for Black patients residing in communities with less than $10 \%$ poverty [3]. Black and Hispanic patients with OA undergoing total hip (THA) and total knee arthroplasty (TKA), when compared to White patients, present with more pain and worse function at baseline [3] and seek arthroplasty with more severe disease [4]. As baseline status is strongly linked to outcomes [5], late presentation may have long-term negative consequences.

For patients with RA, therapy utilization, disease activity, and health-related quality of life are also linked to race, socioeconomic status, and educational attainment [6, 7]. Similarly, Black and Hispanic patients with RA who received ongoing care for their disease in community-based practices have previously been shown to have worse pain and function and higher disease activity scores compared to White patients [8].

It is not known if Black or Hispanic patients with RA undergoing arthroplasty have more pain, worse function, and higher disease activity at the time of THA and TKA compared to White patients with RA. The objective of this study was to assess the pain, function, and disease activity status of Black and Hispanic patients with RA at the time of presentation for elective THA and TKA. We hypothesized that patients with RA who are Black or Hispanic, similar to Black or Hispanic patients with osteoarthritis (OA), would have worse pain [9] and function [10] at the time of THA and TKA, and that higher educational attainment would mitigate the effect of race.

\section{Methods}

\section{Study design and setting}

This is a cross-sectional cohort study using data acquired prospectively from October 2013 through November 2018. Black, White, and Hispanic patients with RA undergoing elective THA and TKA at a single, highvolume tertiary care center for musculoskeletal diseases were recruited prior to surgery and underwent a comprehensive evaluation, including patient- and physicianreported measures and laboratory evaluations performed pre-operatively. Confirmed cases were included in the study after informed consent was obtained.

\section{Participants}

Patients undergoing elective THA or TKA who were $\geq 18$ were screened via electronic medical record to identify patients with RA. Patients were included if they met American College of Rheumatology/European League Against Rheumatism (ACR/EULAR) 2010 or 1987 criteria [11] or if the diagnosis was confirmed by the principal investigator (SG). We excluded patients with crystalline arthropathy or other rheumatic diseases including systemic lupus erythematosus, ankylosing spondylitis, and psoriatic arthritis, those who were unable to understand or read English, or those deemed unreliable to follow the study protocol.

\section{Exposure and outcomes}

The exposure was minority status (self identified as Black or Hispanic) among patients with RA undergoing arthroplasty. The outcomes of interest in our study were pre-operative function [the Multidimensional Health Assessment Questionnaire (MDHAQ)], pain [the Visual Analogue Scale (VAS)], and disease activity [the Disease Activity Score (DAS28)]. The exposure and outcomes were measured pre-operatively.

\section{Data collection}

Baseline data collected included age, sex, education level, and medications. We grouped patients into minority and non-minority cohorts based on self-identification. The minority group included Hispanic, Black or African American, and mixed race patients; the non-minority group were White patients. Poverty at the community level was determined using census tract data, by linking individual patients' addresses via geocoding to their respective census tracts. We obtained census tract-level socioeconomic variables from the American Community Survey/United States Census using the Arc Geographic Information Systems. We used 20\% of the population living below poverty as the point below which health impacts are reported $[12,13]$. The census tract variable of interest was "percentage of families and people whose income in the last 12 months is below the poverty level All families".

We assessed patients using patient and provider global assessments as well as tender and swollen joint counts. Inflammatory marker measurement including erythrocyte sedimentation rate (ESR) and C-reactive protein (CRP), rheumatoid factor (RF) and anti-citrullinated protein antibody (ACPA) were obtained within two weeks prior to surgery or on the day of surgery, prior to the surgical incision.

Medication use was per standard of care and was recorded. Biologic usage at the time of surgery was captured and analyzed as a binary categorical variable. Patients who discontinued their biologics for the surgery 
were considered to be taking biologics. Insurance status included Medicaid, Medicare, or commercial. Medicaid is a public assistance program providing insurance to those in need based on income, and Medicare is an agebased federal insurance program provided to people above age 65 as well as those with disabilities. Medicaid insurance status was used as a proxy for income at the individual level, given the inaccuracies of self-reported income assessments [14]. Those presenting with two types of insurance were categorized into one of the existing insurance status categories according to the following priority: Medicaid followed by Medicare and finally commercial insurances. Patients were marked as having one type of insurance in these cases. For example, a patient who had both Medicare and commercial insurance was marked as having Medicare. Education was recorded as a binary categorical variable with those achieving some college or greater differentiated from those who achieved high school or less.

Function was assessed using the validated MDHAQ, which provides patients with specific questions (example: ability to get in and out of bed) in an effort to assess their overall functional status. The score is comprised of 10 activities of daily living which are scored from 0 to 3 with a score of 0 implying no difficulty performing the activity and 3 implying an inability to perform the activity. The score total is $0-30$; a higher number indicates a worse functional status [15]. Pain was measured using the visual analogue scale (VAS) pain score, which is a user friendly scoring system where patients indicate their level of pain on a spectrum from 1 to 10 , with 10 being unbearable pain [16]. Both MDHAQ and VAS were recorded as continuous variables. The DAS-28 [17] is a composite measure of disease activity that includes swollen and tender joint counts performed by the physician, plus the ESR and the patient's global assessment of their health (PGA) [18]. A higher DAS-28 score implies a more active disease state. DAS28-ESR was chosen as the primary outcome of interest and as the measure of disease activity; it was binarized to patients with moderate or high disease activity (DAS28 $\geq 3.2$ ) and those with low disease activity or remission of disease (DAS28 $<3.2$ ).

\section{Statistical analysis}

The distribution of continuous baseline patient characteristics was assessed for normality using the ShapiroWilk test. Normally distributed values were summarized as mean $\pm \mathrm{SD}$ and compared using $\mathrm{t}$-tests. If nonnormally distributed, values were reported as median [25th percentile, 75th percentile] and compared using the Wilcoxon rank-sum test. Categorical variables were summarized as frequency and percent and compared across minority status using Fisher's exact tests. Student t-test or the Wilcoxon rank-sum test were used, as appropriate, to compare continuous variables across minority status.

Univariate and multivariable linear regressions were performed to assess the impact of minority status on preoperative pain (VAS score), and function (MDHAQ), while univariate and multivariable logistic regressions were performed to assess odds of moderate/severe RA disease activity (DAS28-ESR). Results of linear models are summarized with slope $(\beta) \pm \mathrm{SE}$, while logistic models are summarized with odds ratios (OR) and 95\% confidence intervals (CI). Mean and Standard Deviation (SD) were used for values that were normally distributed and median and Interquartile Range (IQR) were used for values that were not normally distributed. The following variables were forced into all multivariable models as they have been recognized as predictors of total joint replacement outcomes in previous studies and are relevant to the research interest: education, insurance status, age, sex, and biologics [19].

\section{Missing data}

For missing data, we retained all observations that recorded race and insurance status in the univariate analyses. In the multivariable model, the maximum number of patients that had data for each variable included in the model were retained i.e. minority status, education, insurance type, age, sex, use of biologics and MDHAQ score were all required in order to be included in the multivariable model for odds of moderate/high DAS-28 ESR (Table 3). We excluded patients with missing values for race and insurance status. One White patient opted not to disclose gender but was included in the analysis. All analyses were performed using SAS version 9.4. A two-tailed $p<0.05$ was considered statistically significant.

This study is reported per the Strengthening the Reporting of Observational Studies in Epidemiology (STROBE) checklist for cohort studies. This study was approved by the hospital institutional review board and all included patients provided informed consent.

\section{Results}

Our cohort included 164 patients with rheumatoid arthritis undergoing elective THA or TKA, of which 37 (22.6\%) were minorities defined as either Black or Hispanic, and 127 (77.4\%) were White patients (Table 1). The mean age was 62.5 , the majority were women (87\%), and most achieved an education level of at least some college $(88 \%)$. There was a trend towards younger age for minority patients, median (IQR): 56.8 (51.068.8), when compared to White patients, median (IQR): 64.0 (55.0-71.5), although this difference was not significant. When the patients were linked to census tracts via 
Table 1 Demographics, overall and by minority status collected pre-operatively

\begin{tabular}{|c|c|c|c|c|}
\hline Variable & $\begin{array}{l}\text { Overall } \\
(N=164)\end{array}$ & $\begin{array}{l}\text { Minority }^{a} \\
(N=37)\end{array}$ & $\begin{array}{l}\text { White } \\
(N=127)\end{array}$ & $p$-value \\
\hline Age, years, median [IQR] & $62.5[54.7,71.2]$ & $56.8[51.0,68.8]$ & $64.0[55.1,71.5]$ & 0.063 \\
\hline Sex, male & $22(13.41 \%)$ & $3(8.11 \%)$ & $19(15.08 \%)$ & 0.41 \\
\hline $\mathrm{BMI}>30$ & 66 (40.24\%) & $26(70.27 \%)$ & 40 (31.50\%) & $<0.0001$ \\
\hline Ever smoker, yes & 87 (53.05\%) & $18(48.65 \%)$ & $69(54.33 \%)$ & 0.55 \\
\hline Education, Graduated HS \& - & $18(11.76 \%)$ & $4(12.90 \%)$ & $14(11.48 \%)$ & 0.76 \\
\hline Some college \& + & $135(88.24 \%)$ & $27(87.10 \%)$ & 108 (88.52\%) & \\
\hline Insurance, N/A & $23(14.11 \%)$ & $6(16.22 \%)$ & $17(13.49 \%)$ & \\
\hline Commercial & $54(33.13 \%)$ & 9 (29.03\%) & $45(41.28 \%)$ & $<0.001$ \\
\hline Medicare & $77(47.24 \%)$ & $13(41.94 \%)$ & $64(58.72 \%)$ & \\
\hline Medicaid & $9(5.52 \%)$ & 9 (29.03\%) & $0(0.0 \%)$ & \\
\hline Biologics, Yes & $90(55.56 \%)$ & $23(62.16 \%)$ & $67(53.60 \%)$ & 0.45 \\
\hline VAS Pain Score, median [IQR] & $6.0[4.0,8.0]$ & $7.5[4.0,8.0]$ & $6.0[4.0,8.0]$ & 0.029 \\
\hline MDHAQ Score, mean \pm SD & $11.8 \pm 5.3$ & $12.3 \pm 5.1$ & $11.6 \pm 5.3$ & 0.53 \\
\hline $\mathrm{DAS} 28-\mathrm{ESR}$, mean $\pm \mathrm{SD}$ & $3.8 \pm 1.3$ & $4.1 \pm 1.3$ & $3.8 \pm 1.2$ & 0.18 \\
\hline
\end{tabular}

${ }^{a}$ Minority status: Black or African American, Hispanic or Mixed

Significant values are bolded. Percentages in parenthesis unless otherwise indicated

IQR Interquartile Range, VAS Visual Analogue Scale, MDHAQ Multidimensional Health Assessment Questionnaire, DAS28-ESR Disease Activity Score28-Erythrocyte Sedimentation Rate, HS High School

geocoding $5(3 \%)$ of the cohort $(n=164)$ lived in census tracts with $20 \%$ or more of the population living below poverty level, $3(60 \%)$ were in the minority group (2 Black, 1 Hispanic), and 2 (40\%) were White.

Most minority patients (87\%) and White patients $(88.5 \%)$ achieved an education level of at least some college, with no significant difference between the groups. The majority of the cohort was on biologics at the time of arthroplasty (56\%); although not significant, a greater percentage of minority patients $(62 \%)$ were on biologics than White patients $(54 \%, p=0.45)$. Insurance type was significantly different between minority and White groups $(p<0.001): 29 \%$ of minority group vs. $42 \%$ of White patients had commercial insurance, $42 \%$ of minority group vs. 59\% in White group had Medicare, 29\% of minority group vs. $0 \%$ of White patients had Medicaid. The median VAS pain score for the overall cohort was 6.0 (IQR; 4.0, 8.0), and was significantly higher among minorities (7.5 vs $6.0, p=0.03$ ). The mean MDHAQ score for the cohort was 11.8 (SD 5.3) and the mean DAS28-ESR score for the cohort was 3.8 (SD 1.), with no significant difference between groups $(p=0.53$ and 0.18 , respectively).

In the univariate analyses to determine predictors of RA disease activity (Table 2) education level and sex were significant predictors of RA disease activity. In our multivariable model (Table 3), MDHAQ score was a significant predictor of moderate/severe RA disease activity $[\mathrm{OR}=1.09 ; 95 \% \mathrm{CI}(1.0,1.17), p=0.04]$. Although higher education level [OR $=9.57$; $95 \% \mathrm{CI}(2.2,42.2), p=0.003$ ] was a statistically significant predictor of moderate/high
RA disease activity, the difference in education between groups was small. Only 18 (11.8\%) patients had an educational level of high school or less across both minority and White groups and the wide confidence intervals for education indicate that this estimate had limited precision.

In the univariate model to determine predictors of VAS pain at the time of presentation for arthroplasty, only MDHAQ $(\beta 0.18 \pm 0.04, p<.0001)$ was a significant predictor of worse pain at the time of arthroplasty (Table 4). In the multivariable model, after forcing variables of interest into the model, only MDHAQ remained

Table 2 Univariate analysis to determine the risk of high RA disease activity (DAS28-ESR,Logistic Regressions)

\begin{tabular}{|c|c|c|}
\hline DAS28-ESR & Odds ratio $(95 \% \mathrm{Cl})$ & $p$-value \\
\hline Minority $(Y e s)^{a}$ & $1.16(0.54,2.49)$ & 0.70 \\
\hline Education (Some college +) & $4.48(1.60,12.57)$ & 0.004 \\
\hline Medicare ${ }^{b}$ & $1.23(0.62,2.42)$ & 0.55 \\
\hline Medicaid $^{b}$ & $1.69(0.39,7.40)$ & 0.49 \\
\hline Age & $0.99(0.96,1.02)$ & 0.38 \\
\hline Sex (Female) & $3.25(1.34,7.86)$ & 0.009 \\
\hline Biologics (Yes) & $1.02(0.54,1.90)$ & 0.97 \\
\hline MDHAQ Score & $1.11(1.04,1.19)$ & 0.003 \\
\hline
\end{tabular}

${ }^{a}$ Minority status: Black or African American, Hispanic or Mixed ${ }^{\mathrm{b}}$ Ref commercial

Significant values are bolded

DAS28-ESR Disease Activity Score28-Erythrocyte Sedimentation Rate, $\beta$ Beta Coefficient

SE Standard Error, MDHAQ Multidimensional Health Assessment Questionnaire, $\mathrm{Cl}$ Confidence Interval 
Table 3 Multivariable analysis to determine the risk of RA disease activity (DAS28-ESR, Generalized Logistic Model)

\begin{tabular}{lll}
\hline Variable & Odds ratio $(95 \% \mathrm{Cl})$ & p-value \\
\hline Minority $^{\mathrm{a}}$ (Yes) & $1.29(0.41,4.05)$ & 0.66 \\
Education (Some college +) $^{\text {Medicaid }}{ }^{\mathrm{b}}$ & $\mathbf{9 . 5 7}(\mathbf{2 . 1 7}, \mathbf{4 2 . 2 1 )}$ & $\mathbf{0 . 0 0 3}$ \\
Medicare $^{\mathrm{b}}$ & $4.30(0.28,66.69)$ & 0.30 \\
Age $_{\text {Sex (Female) }}$ & $1.48(0.49,4.45)$ & 0.48 \\
Biologics (Yes) $_{\text {MDHAQ Score }}$ & $0.97(0.92,1.02)$ & 0.22 \\
& $2.35(0.74,7.49)$ & 0.15 \\
& $0.64(0.27,1.48)$ & 0.30 \\
& $\mathbf{1 . 0 9}(\mathbf{1 . 0 0 , 1 . 1 7 )}$ & $\mathbf{0 . 0 4 4}$
\end{tabular}

${ }^{\mathrm{a} M i n o r i t y ~ s t a t u s: ~ B l a c k}$ or African American, Hispanic or Mixed

${ }^{\text {b Ref: Commercial }}$

Significant values are bolded

DAS28-ESR Disease Activity Score28-Erythrocyte Sedimentation Rate, $\beta$ Beta Coefficient, SE Standard Error, MDHAQ Multidimensional Health Assessment Questionnaire, $\mathrm{Cl}$ Confidence Interval

a significant predictor of worse pain scores $(\beta 0.17+/-$ $0.05, p=0.0002$ ) (Table 5).

In our univariate model to explore predictors of MDHAQ at the time of arthroplasty, we found no statistically significant predictive factors (Table 6). In our multivariable model after forcing in factors of interest, only age was a predictor of MDHAQ at the time of $\operatorname{arthroplasty}(\beta-0.17+/-0.06, p=0.004)$ (Table 7). Neither minority status nor Medicaid insurance were significant in any of our models to predict the status of our patients with RA at the time they seek arthroplasty.

\section{Discussion}

In this study we assess whether patients with RA who are Black or Hispanic have worse pain, function and disease activity at the time of arthroplasty and find no difference when compared to White patients with RA, unlike the experience of Black patients with osteoarthritis (OA) who present for arthroplasty with worse pain

Table 4 Univariate analysis to determine the risk of pain (VAS Pain Score, Simple Linear Regression)

\begin{tabular}{lll}
\hline Variable & $\beta \pm \mathrm{SE}$ & $p$-value \\
\hline Minority $^{\mathrm{a}}$ (Yes) & $1.01 \pm 0.54$ & 0.06 \\
Education (Some college + ) $^{\text {Medicare }}{ }^{\mathrm{b}}$ & $0.66 \pm 0.68$ & 0.33 \\
Medicaid $^{\mathrm{b}}$ & $0.46 \pm 0.51$ & 0.36 \\
Age & $-0.13 \pm 1.17$ & 0.91 \\
Sex (Female) & $-0.01 \pm 0.19$ & 0.70 \\
Biologics (Yes) $_{\text {MDHAQ Score }}$ & $0.62 \pm 0.65$ & 0.34 \\
& $0.56 \pm 0.45$ & 0.21 \\
\hline
\end{tabular}

${ }^{a}$ Minority status: Black or African American, Hispanic or Mixed

${ }^{\mathrm{b}}$ Ref commercial; see below for minority status definition

Significant values are bolded

VAS Visual Analogue Scale, $\beta$ Beta Coefficient, SE Standard Error, MDHAQ

Multidimensional Health Assessment Questionnaire
Table 5 Multivariable analysis to determine risk of pain (VAS Pain Score, Generalized Linear Model)

\begin{tabular}{lll}
\hline VAS Pain Score & $\beta \pm \mathrm{SE}$ & $p$-value \\
\hline Minority $^{\mathrm{a}}$ (Yes) & $0.87 \pm 0.66$ & 0.18 \\
Education (Some college +) & $0.12 \pm 0.77$ & 0.87 \\
Medicaid $^{b}$ & $-0.13 \pm 1.37$ & 0.93 \\
Medicare $^{\mathrm{b}}$ & $0.22 \pm 0.67$ & 0.75 \\
Age & $0.02 \pm 0.03$ & 0.44 \\
Sex (Female) & $0.16 \pm 0.70$ & 0.82 \\
Biologics (Yes) $_{\text {MDHAQ Score }}$ & $0.81 \pm 0.50$ & 0.11 \\
& $\mathbf{0 . 1 7} \pm \mathbf{0 . 0 5}$ & $\mathbf{0 . 0 0 0 2}$
\end{tabular}

${ }^{a}$ Minority status: Black or African American, Hispanic or Mixed

${ }^{\mathrm{b}}$ Ref: Commercial

Significant values are bolded

VAS Visual Analogue Scale, $\beta$ Beta Coefficient, SE Standard Error, MDHAQ

Multidimensional Health Assessment Questionnaire

and function [20]. For Black and Hispanic patients with RA undergoing arthroplasty at a high-volume surgical hospital with high levels of education, who live in communities with little poverty, there is no difference in baseline pain or function compared to White patients. Similarly, disease activity and biologics use were no different between the groups.

Socioeconomic factors such as poverty and lower educational attainment are disproportionately seen among Black and Hispanic patients who are more likely to live in poor communities where health outcomes are poorer, so the contributions of "place vs. race" can be hard to discern [4]. Using geocoding and linking our cohort to individual census tracts, we demonstrated that there was little difference in this study in the proportion of residence in a census tract below the poverty line across minority status. Although Medicaid insurance, a reliable surrogate for individual level poverty, was significantly different between the groups in this cohort, educational levels and community resources were equivalent, and all received care in the same high-volume hospital. In this

Table 6 Univariate analysis to determine the risk of poor function y (MDHAQ Score,Simple Linear Regressions)

\begin{tabular}{lll}
\hline MDHAQ Score & $\beta \pm$ SE & $p$-value \\
\hline Minority $^{\text {a }}$ (Yes) & $0.66 \pm 1.03$ & 0.53 \\
Education (Some college +) $^{\text {Medicare }}{ }^{b}$ & $-0.19 \pm 1.27$ & 0.88 \\
Medicaid $^{b}$ & $0.12 \pm 0.97$ & 0.90 \\
Age $_{\text {Sex (Female) }}$ & $0.71 \pm 2.15$ & 0.74 \\
Biologics (Yes) $^{\text {Minority Stus: Black or African American }}$ & $-0.06 \pm 0.04$ & 0.065 \\
\hline
\end{tabular}

${ }^{a}$ Minority status: Black or African American, Hispanic or Mixed

${ }^{b}$ Ref commercial; see below for minority status definition

Significant values are bolded

MDHAQ Multidimensional Health Assessment Questionnaire, $\beta$ Beta Coefficient, SE Standard Error 
Table 7 Multivariable analysis to determine risk of poor function (MDHAQ Score, Generalized Linear Model)

\begin{tabular}{lll}
\hline MDHAQ Score & $\beta \pm$ SE & $p$-value \\
\hline Minority $^{\mathrm{a}}$ (Yes) & $1.44 \pm 1.27$ & 0.26 \\
Education (Some college +) $^{\text {Medicaid }}{ }^{\mathrm{b}}$ & $-0.24 \pm 1.49$ & 0.87 \\
Medicare $^{\mathrm{b}}$ & $-2.60 \pm 2.66$ & 0.33 \\
Age & $2.31 \pm 1.28$ & 0.07 \\
Sex (Female) & $\mathbf{- 0 . 1 7} \pm \mathbf{0 . 0 6}$ & $\mathbf{0 . 0 0 4}$ \\
Biologics (Yes) & $0.26 \pm 1.37$ & 0.85 \\
\end{tabular}

${ }^{a}$ Minority status: Black or African American, Hispanic or Mixed

${ }^{b}$ Ref: Commercial

Significant values are bolded

MDHAQ Multidimensional Health Assessment Questionnaire, $\beta$ Beta Coefficient, SE Standard Error

cohort, race and ethnicity are not associated with worse disease status at the time of arthroplasty.

Median household income is used to determine gradients in community poverty and offer a different perspective than using individual income [21]. In this study, while there was significantly more poverty at the individual level (Medicaid insurance) for minority patients, few in the cohort lived in poor communities and there was little difference in community level poverty between the groups.

Biologic disease modifying antirheumatic drugs (DMARDs) may be underutilized which may contribute to poorer disease status in Black patients with RA who have Medicare [22] and Medicaid [23] insurance. Black patients with the California Medicaid program (Medical), seen in outpatient and inpatient settings are reported to underutilize biologics when compared to White patients, and utilize DMARDs 6-11\% less than other racial groups [24]. We demonstrate that in minority patients with long standing RA seen prior to arthroplasty, some of whom are insured by Medicaid, the utilization of biologic DMARDs is no different compared to White patients with RA with $56 \%$ of both groups using biologics. RA patients undergoing arthroplasty may represent a cohort of patients who are selecting more aggressive care overall, including biologics, as compared to other RA patients. Alternatively, the similarities in education and community poverty level may be mitigating the disparities described previously in utilization of therapies for patients with RA.

Several limitations regarding this study should be noted. While minority status was not found to be a significant predictor of any RA disease outcomes, this may be due to the characteristics of the present cohort. The cohort consisted of majority White females with some college education or higher, and we have previously demonstrated that education can mitigate the effects of poverty on arthroplasty outcomes, but in this study, both white and minority patients had similar education, limiting the potential for introducing bias [2]. As most patients receive care in community hospitals where a broader range of education and individual level income exists our results may not be generalizable. The study cohort is limited to patients who present for arthroplasty at a tertiary care specialty hospital in New York City, another limitation in generalizability. Racial or ethnic differences in pain perception or impact could bias these results; however, little difference in pain perception has been found between Blacks, Whites, or Hispanics [25]. Finally, as in any observational study, our results may be affected by unmeasured confounders.

Future studies would benefit from examination of this cohort of patients in the post-operative period where THA and TKA outcomes would be studied independently. Pre-operative factors such as radiographic severity, pain perception and catastrophizing as well as hip disability and osteoarthritis outcome score/knee injury and osteoarthritis outcome score (HOOS/KOOS) pain and function should be examined to assess the impact on the post-operative outcomes and post procedure complications.

\section{Conclusion}

In summary, in a cohort of highly educated patients with RA receiving care at a high volume academic surgical hospital, residing in communities with low levels of poverty despite significant poverty at the individual level, we find that Black and Hispanic patients with RA do not have worse pain, function, or disease activity at the time of arthroplasty. There was no difference in biologic usage across minority status. Our findings lend further support to the concept of "place, not race" in the study of health care disparities.

\section{Abbreviations}

ACPA: Anti-Citrullinated Protein Antibody; ACR/EULAR: American College of Rheumatology/European League Against Rheumatism; CRP: C-Reactive Protein; DAS28-ESR: Disease Activity Score-Erythrocyte Sedimentation Rate; DMARD: Disease Modifying Antirheumatic Drugs; ESR: Erythrocyte Sedimentation Rate; IQR: Interquartile Range; MDHAQ: Multidimensional Health Assessment Questionnaire; Medical: California Medicaid Program; OA: Osteoarthritis; OR: Odds Ratio; PGA: Patient's Global Assessment; RA: Rheumatoid Arthritis; SAS: Statistical Analysis System; SD: Standard Deviation; STROBE: Strengthening the Reporting of Observational Studies in Epidemiology; THA: Total Hip Arthroplasty; TKA: Total Knee Arthroplasty; VAS: Visual Analogue Scale; $\beta$ : Beta Coefficient of Slope

\section{Acknowledgements}

I.N.-M. is funded by a National Institute of Arthritis and Musculoskeletal and Skin Disease K23 grant (K23-AR068449).

Diyu Pearce-Fisher contributed to the preparation of the manuscript.

\section{Author's contributions}

J.H., B.M., and S.G. contributed to the writing of the manuscript, concept design, and interpretation of data. J.F. analyzed and interpreted the data. I.N. M. and L.R. contributed to the study planning and design and the manuscript preparation. C.B. and S.M. contributed to the acquisition of specimens, study planning, and manuscript preparation. M.F. contributed to 
the acquisition of specimens and manuscript preparation. M.P. contributed to the study design and manuscript preparation. D.O. contributed to the acquisition of specimens, study design, and interpretation of results. All authors read and approved the final manuscript.

\section{Funding}

No funding.

\section{Availability of data and materials}

The datasets used and/or analyzed during the current study are available upon reasonable request.

\section{Ethics approval and consent to participate}

Ethics approval was obtained from the Hospital for Special Surgery (IRB\#: 2014-233). Written informed consent for participation in the study was obtained from all participants.

\section{Consent for publication}

Not applicable.

\section{Competing interests}

J.H., B.M., J.F., I.N.-M., C. B, S.M., M.F., M.P., L.R., and D.O. declare that they have no competing interests in relation to the work. S.G. reports personal fees from Current Rheumatology reports as section editor and non-financial support from BMC Rheumatology as section editor.

\section{Author details}

${ }^{1}$ Mount Sinai St. Luke's-West, New York, NY, USA. ${ }^{2}$ Hospital for Special Surgery, 535 E 70th Street, New York, NY 10021, USA. ${ }^{3}$ Weill Cornell Medicine, New York, NY, USA. ${ }^{4}$ The Rockefeller University, New York, NY, USA.

Received: 9 July 2019 Accepted: 30 January 2020

Published online: 29 February 2020

\section{References}

1. Shahid H, Singh J. Racial/Ethnic Disparity in Rates and Outcomes of Total Joint Arthroplasty. Curr Rheumatol Rep 2016; Apr;18(4):1-13.

2. Goodman SM, Mandl LA, Mehta B, Navarro-Millan I, Russell LA, Parks ML, et al. Does Education Level Mitigate the Effect of Poverty on Total Knee Arthroplasty Outcomes?. Arthritis Care \& Research 2018; Jun;70(6):884-91.

3. Goodman SM, Mandl LA, Parks ML, Zhang M, McHugh KR, Lee YY, et al. Disparities in TKA Outcomes: Census Tract Data Show Interactions Between Race and Poverty. Clin Orthop Relat Res 2016; Sep;474(9):1986-95.

4. Goodman S, Mehta B, Zhang M, Szymonifka J, Nguyen J, Lee L, et al. Disparities in Total Hip Arthroplasty Outcomes: Census Tract Data Show Interactions Between Race and Community Deprivation. Journal of the American Academy of Orthopaedic Surgeons 2018; Nov 1;26(21):e464.

5. Jeremy Holtzman, Khal Saleh, Robert Kane. EFFECT OF BASELINE FUNCTIONAL STATUS AND PAIN ON OUTCOMES OF TOTAL HIP ARTH ROPLASTY. Journal of Bone and Joint Surgery 2002; Nov 1;84(11):1942-8.

6. Molina E, del Rincon I, Restrepo JF, Battafarano DF, Escalante A. Association of Socioeconomic Status With Treatment Delays, Disease Activity, Joint Damage, and Disability in Rheumatoid Arthritis. Arthritis Care \& Research 2015; Jul;67(7):940-6.

7. Baldassari AR, Cleveland RJ, Jonas BL, Conn DL, Moreland LW, Bridges SL, et al. Socioeconomic Disparities in the Health of African Americans With Rheumatoid Arthritis From the Southeastern United States. Arthritis Care \& Research 2014; Dec;66(12):1808-17.

8. Greenberg JD, Spruill TM, Shan Y, Reed G, Kremer JM, Potter J, et al. Racial and ethnic disparities in disease activity in patients with rheumatoid arthritis. Am J Med 2013; Dec;126(12):1089-98.

9. Hausmann LRM, Brandt CA, Carroll CM, Fenton BT, Ibrahim SA, Becker WC, et al. Racial and Ethnic Differences in Total Knee Arthroplasty in the Veterans Affairs Health Care System, 2001-2013. Arthritis Care \& Research 2017; Aug;69(8):1171-1178.

10. Allen, K.D.|Helmick, C.G.|Schwartz, T.A.|DeVellis, R.F.|Renner, J.B.|Jordan, J.M. Racial differences in self-reported pain and function among individuals with radiographic hip and knee osteoarthritis: the Johnston County osteoarthritis project. Osteoarthr Cartil 2009;17(9):1132-1136.

11. Kasturi S, Goldstein B, Malspeis S, Karlson E, Costenbader K. Comparison of the 1987 American College of Rheumatology and the 2010 American
College of Rheumatology/European League against Rheumatism criteria for classification of rheumatoid arthritis in the Nurses' Health Study cohorts. Rheumatol Int 2014; Mar;34(3):407-11.

12. Krieger N, Chen JT, Waterman PD, Rehkopf DH, Subramanian SV. Painting a truer picture of US socioeconomic and racial/ethnic health inequalities: the Public Health Disparities Geocoding Project. Am J Public Health 2005; Feb; 95(2):312-23.

13. Krieger N, Chen JT, Waterman PD, Rehkopf DH, Subramanian SV. Race/ ethnicity, gender, and monitoring socioeconomic gradients in health: a comparison of area-based socioeconomic measures--the public health disparities geocoding project. Am J Public Health 2003; Oct;93(10):1655-71.

14. Foraker RE, Rose KM, Whitsel EA, Suchindran CM, Wood JL, Rosamond WD. Neighborhood socioeconomic status, Medicaid coverage and medical management of myocardial infarction: atherosclerosis risk in communities (ARIC) community surveillance. BMC Public Health 2010; Oct 21;10:632.

15. Pincus $T$, Swearingen $C$, Wolfe F. Toward a multidimensional Health Assessment Questionnaire (MDHAQ): assessment of advanced activities of daily living and psychological status in the patient-friendly health assessment questionnaire format. Arthritis and Rheumatism 1999; Oct;42(10): 2220-30.

16. Boonstra AM, Schiphorst Preuper HR, Reneman MF, Posthumus JB, Stewart RE. Reliability and validity of the visual analogue scale for disability in patients with chronic musculoskeletal pain. International Journal of Rehabilitation Research 2008; Jun;31(2):165-9.

17. THEODORE PINCUS, CHRISTOPHER J. SWEARINGEN, MARTIN BERGMAN, YUSUF YAZICI. RAPID3 (Routine Assessment of Patient Index Data 3), a Rheumatoid Arthritis Index Without Formal Joint Counts for Routine Care: Proposed Severity Categories Compared to Disease Activity Score and Clinical Disease Activity Index Categories. The Journal of Rheumatology 2008; Nov 1;35(11):2136-47.

18. Wells G, Becker JC, Teng J, Dougados M, Schiff M, Smolen J, et al. Validation of the 28-joint disease activity score (DAS28) and European league against rheumatism response criteria based on C-reactive protein against disease progression in patients with rheumatoid arthritis, and comparison with the DAS28 based on erythrocyte sedimentation rate. Ann Rheum Dis. 2009; 68(6):954-60.

19. Stefanie Hofstede, Maaike Gademan, Thea Vliet Vlieland, Rob Nelissen, Perla Marang-van de Mheen. Preoperative predictors for outcomes after total hip replacement in patients with osteoarthritis: a systematic review 2016;

20. Mehta BY, Bass AR, Goto R, Russell LA, Parks ML, Figgie MP, et al. Disparities in Outcomes for Blacks versus Whites Undergoing Total Hip Arthroplasty: A Systematic Literature Review. J Rheumatol 2018; May;45(5):717-22.

21. Krieger N, Chen JT, Waterman PD, Soobader MJ, Subramanian SV, Carson R. Choosing area based socioeconomic measures to monitor social inequalities in low birth weight and childhood lead poisoning: The Public Health Disparities Geocoding Project (US). J Epidemiol Community Health 2003; Mar;57(3):186-99.

22. Yazdany J, Dudley RA, Chen R, Lin GA, Tseng C. Coverage for High-Cost Specialty Drugs for Rheumatoid Arthritis in Medicare Part D. Arthritis \& Rheumatology 2015; Jun;67(6):1474-80

23. Bonafede M, Joseph GJ, Shah N, Princic N, Harrison DJ. Cost of tumor necrosis factor blockers per patient with rheumatoid arthritis in a multistate Medicaid population. ClinicoEconomics and Outcomes Research : CEOR. 2014;6:381-8.

24. Chu LH, Portugal C, Kawatkar AA, Stohl W, Nichol MB. Racial/ethnic differences in the use of biologic disease-modifying antirheumatic drugs among California Medicaid rheumatoid arthritis patients. Arthritis Care Res (Hoboken) 2013; Feb;65(2):299-303.

25. Edwards RR, Moric M, Husfeldt B, Buvanendran A, Ivankovich O. Ethnic similarities and differences in the chronic pain experience: a comparison of African American, Hispanic, and white patients. Pain Med. 2005 Jan;6(1):88-98.

\section{Publisher's Note}

Springer Nature remains neutral with regard to jurisdictional claims in published maps and institutional affiliations. 